\title{
Comparison of video based learning and lecture based learning in last year medical faculty students
}

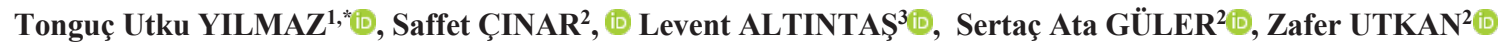 \\ ${ }^{1}$ Atakent Hospital Organ Transplantation Unit, Acibadem Mehmet Ali Aydınlar University, İstanbul, Turkey \\ ${ }^{2}$ Department of General Surgery, Faculty of Medicine, Kocaeli University, Kocaeli, Turkey \\ ${ }^{3}$ Department of Medical Education, Faculty of Medicine, Acibadem Mehmet Ali Aydınlar University, İstanbul, Turkey
}

\begin{abstract}
\begin{tabular}{ccccc}
\hline Received: 07.09 .2020 & $\bullet$ & Accepted/Published Online: 16.12 .2020 & $\bullet$ & Final Version: 14.03 .2021 \\
\hline
\end{tabular}
Abstract

Cats are the main host of Toxoplasma gondii and pregnant women who own cats at home may be at risk. This condition may cause anxiety and stress in pregnant women. The aim of this study is to evaluate toxoplasmosis risk on anxiety and perception of cat owner pregnant women. The study group consisted of 59 pregnant women feeding cats in the home and 369 pregnant women not feeding cats. A Health Anxiety Inventory (HAI-18) was used to measure the anxiety of all participants. Age and anxiety variables were analyzed using a two-sample independent t-test. Gravida, education, and job status were analyzed using chi-square tests, and live to abort ratio \& pregnancy number were analyzed using Fisher exact test. Measurement of the anxiety level between the two groups showed that anxiety is significantly higher among the pregnant women who feed cats in their house with an average of $32.03 \pm 8.72$. The average anxiety level among the pregnant women who don't have cats in their house was $25.94 \pm 8.99$. The difference between the Health Anxiety Inventory of the two groups was significant ( $p=0.0001)$ It was shown in the literature that pets can reduce anxiety but not studied in pregnant women, yet. Our results showed that pregnant women who own cats at home had more environmental anxiety than women who don't feed cats at home which is possibly associated with fear of Toxoplasma gondii transmission. This anxiety may also cause unfavorable pregnancy outcomes like eclampsia, preterm birth, low birth weight and high incidence of cesarean delivery.
\end{abstract}

Keywords: Medical education, video-based learning, knot tying, thyroid examination, covid-19

\section{Introduction}

Clinical skill is a basic requirement of all medical students. Techniques of physical examination in early years of clinics has great influence on subsequent clinical development. Although it's very important, this is left to students who are themselves in the progress of improving their skills. It's a pity that last year medical students were unable to perform exact examination methods very well. As most of the wards spent little time on teaching skill for the last year students, they are unable to practice. As the number of medical students are increasing, the students are staying back for practice. Lack of enough educators and so many works in daily practice led limited time for the students. The subjects of the physical examinations depend on the hospitalized patients. On the other hand, lecture-based learning is not suitable for last year students as the main aim of last year is to practice. Video has the advantages of offering media-rich and visual stimulation covering wide spectrum of the learning styles. Video has the ability to be forwarded, rewound and repeated. Also, it has so many scenarios for the viewers to see the demonstrations of a live process or clinical skills. COVID19 pandemic showed that e-learning can take important part of education. Elearning or video-based learning can be used widely in future.

Here, we want to compare the results of lecture based and video-based learning about thyroid physical examination and knot tying in last year medical students.

\section{Materials and methods}

Medical school is six years and students have rotation in general surgery department on the fourth year. During this rotation, students take lectures and making practices about physical examination of organ systems and knot tying. In the last year, students spent 1 month in general surgery and take part in the diagnosis, treatment and care of the patients. During this one-month period, lessons about physical 
examination, common surgical problems like thyroid diseases, breast diseases, acute abdomen, proctological diseases, approach to trauma and fluid electrolyte disturbances are given. Every student is sharing the treatment responsibility of a patient under the supervision of an instructor. The study was performed among last year medical faculty students called interns who came to general surgery department.

During six-month period, every month the interns who came to general surgery department divided into two groups; video-based group and lecture-based group. Student underwent an exam about physical examination of thyroid and knot tying on the first day of their rotation. During physical examination of thyroid, the instructor observed the students whether they have performed the important steps of thyroid physical examination (Table 1). Total scores were given as percentages (score of the student/30*100). These steps are designed according to the education program of Kocaeli University School of Medicine Physical Examination program (www.tip.kocaeli.edu.tr/muayene_Rehberi.php). Also, the interns were observed whether they can tie a surgical knot. The physical examination is scored according the achievements. Knot tying was successful or unsuccessful. After then the students were randomly grouped into videobased and lecture-based groups. Video-based groups watched a video about thyroid physical examination in which the important steps as in Table 1 had been shown. Also, they watched a video about knot tying. Student were free to stop, undo, or rewind the video. Students in lecture-based group were given lecture about thyroid physical examination in a classroom. Instructor showed the thyroid physical examination steps on one of the students.

\section{Results}

Instructor told the important steps of thyroid examination as mentioned in Table 1. The student was free to ask questions. At least one week after the lessons, the students were undergoing physical examination and knot tying exam again. The pre-lecture and post-lecture exam results were compared with student $\mathrm{t}$ test. $\mathrm{p}<0.05$ was accepted as significant. Also, at the end of post-lecture exam the students were asked whether they felt excited, confident and qualified enough.

Total 42 students included to the study. Every group composed of 21 students. When we compare the thyroid physical examination; there was a significant difference between the pre-lecture scores of both groups and post-lecture scores of the both groups $(\mathrm{P}=0.001)$ (Figure 1). On the other hand, there's not a significant difference between the prelecture scores and post-lecture results of the both groups ( $>0.05$ ) (Fig. 1). When we look at the ability of knot tying; one student from both groups were able to tie knots. After the lecture the number of knots tying ability significantly increased $(\mathrm{p}<0.05)$ (Fig. 1). When we look at the feelings of students, nearly all of the students in both groups felts their self-qualified and confident about the thyroid examination. However, the feeling of excitement ratio in both groups were lower but no significant difference was seen between groups $(\mathrm{p}=0.1)$ (Fig. 2).

Table 1. Thyroid Physical Examination Guide for Medical Faculty Grade 4 Students

Thyroid Physical Examination Steps Scores

1 2

\section{Hand Wash}

Giving information about the examination to the patient

Patient should be seated in a comfortable position with neck in a neutral or slightly extended position

Inspection of thyroid gland from anteriod and lateral approach; mass, symmetry, scars, movement of thyroid gland during swallowing fluid, tongue protrusion

Palpation of thyroid gland:

Location of thyroid isthmus and cricoid cartilage

Retraction sternocleidomastoid muscle and palpate each thyroid lobe. Size, symmetry, consistency, masses, tenderness, palpable thrill

If nodule: size, location, shape, consistency, mobility

Palpate the thyroid during swallowing

Palpate during tongue protrusion

Palpation of neck lymph nodes

Auscultation of the thyroid gland; existence of bruit

Percussion of the sternum

Explain the patient that the examination is finished

Wash hands

Summaries the findings to the instructor

\section{Discussion}

The place of the internet in our lives is an indisputable fact. Given the rise of watching videos from the internet, the share of video management in the transfer of information to people is increasing day by day. With all these developments, the use of internet is rapidly taking place in the medical field. The increasing number of students in medical schools reduces the quality and accessibility of education. Video-based education models are developed to improve the quality of education. Video-based education is used in many fields in medical education practice today (Edwards et al., 1985; Fabius et al., 1994; Fried et al., 2004; Seymour et al., 2002; Summers et al., 1995). The integration of technology in medical education is 
increasing day by day, and studies show that students welcome computerized education positively (Prystowsky et al., 1996; Pusic, 1998). In a study by Lynch et al., a significant increase was noted in the pretest and posttest evaluations made with the students' video education (Lynch et al., 2001). The most important advantage of this training is that video-based education is easy to access by students and there is no time limit to watch the video over and over again. The ability to adjust the speed of education is an additive for poor students. In another study, Greenhalgh, in his comprehensive review on computer assisted learning in medical education, discussed potential advantages for this method of education (Greenhalgh, 2001). It was stated that video education is particularly suitable for subjects such as anatomy and surgery that are visually intense and difficult to conceptualize. With these features, video-based education has become compulsory in many faculties and it has become a condition to pass the class. For last year students which had completed their primary examination educations, video-based education might be helpful for recalling the knowledge. On the other hand, knot tying is an example of skill practice. It was seen in this study that although the knot tying lesson were given on the fourth year, unused skills can be forgotten. However, by giving video-based lectures, these knowledges can be easily remembered. This study never suggests videobased lectures for primary education method, but advice for last year students.

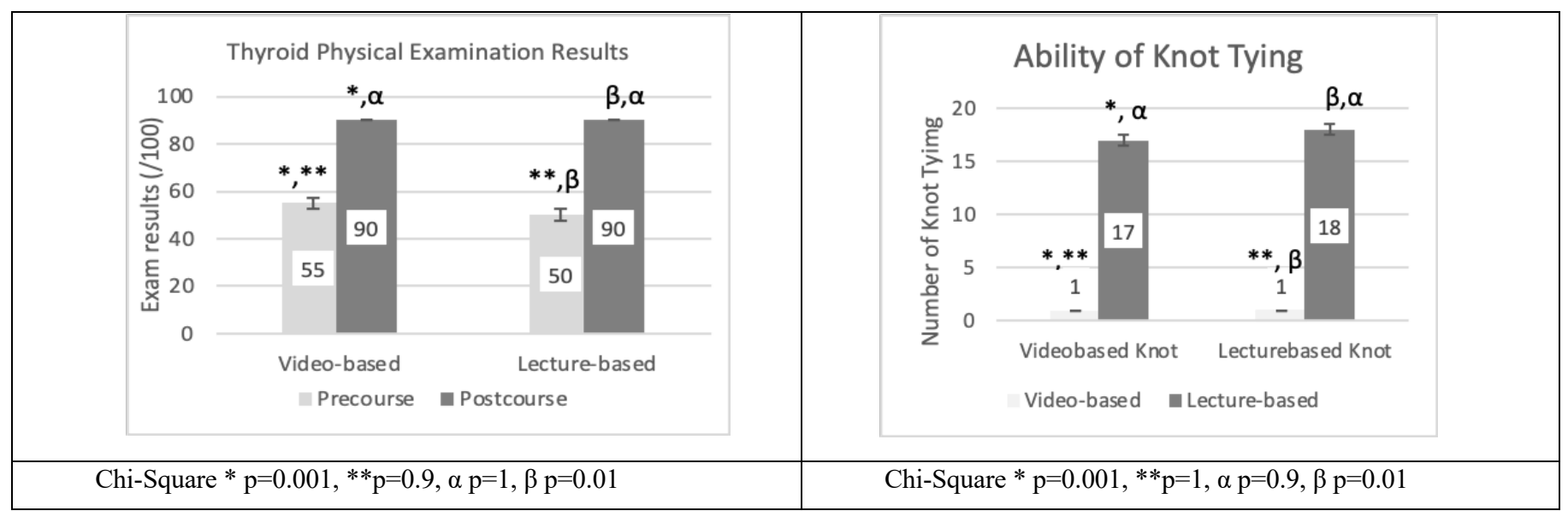

Fig. 1. Comparision of precourse and postcourse thyroid examination results and knot tying ability of video-based and Lecture-based learning group

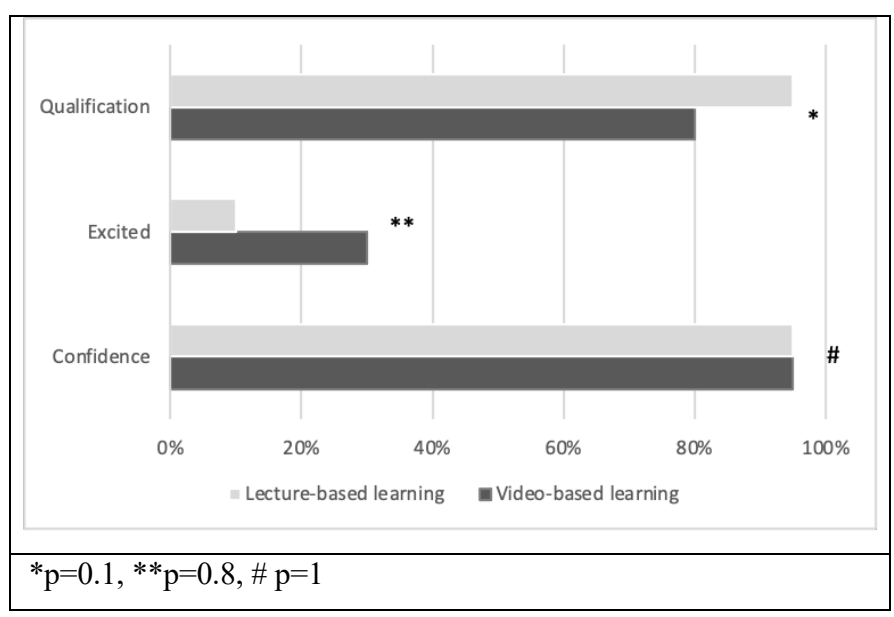

Fig. 2. Comparison of feelings of student in lecture-based and videobased learning groups

It is an indispensable fact for many authorities that the "One to One" apprenticeship model for surgical education will remain the gold standard method. However, new technological applications in education can enable students to learn concepts and techniques more effectively and efficiently. In the study of Summers et al, it was observed that computer assisted learning programs can be effective teaching tools for simple operative skills (Summers et al., 1999). Video education can be a part of treatment not only for students but also for patients. While the importance of traditional physician-patient relationship is still unquestionable, the contribution of video education in terms of gaining knowledge has been demonstrated by studies (Armstrong et al., 2010; Armstrong et al., 2011; Orringer et al., 2005; Shukla et al., 2012). Future studies will be helpful to clearly measure patient satisfaction of video training. Speaking of the positive aspects of video-based education, one should not forget that it is a costly technology (Keane et al., 1991). In the face of this problem, the positive aspects of video-based education should be increased. In particular, the increasing use of it for practical information as well as theoretical knowledge is important for ignoring the high-cost problem. Curriculums supported by computer-based education applications may require less face-to-face lessons, providing training for both experts and students in minimal physical conditions when web-based, and the fact that computer education can be customized, modified, and upgraded is a positive factor in evaluating cost effectiveness.

In the studies conducted, different results were revealed on the contribution of experts in video-based education. Studies conducted by Schmidt and Bjork and Schmidt et al on feedback in motor learning revealed that two groups of students receiving different feedback methods showed better improvement in the coming period, although the students 
showed similar learning progress at the beginning (Schmidt et al., 1989; Schmidt et al., 1992). This result revealed the importance of expert supervision in video-based education. In another study, the importance of expert supervision in videobased education has been shown (Satava, 1993). In response to these results, in a study by Nousiainen et al., They showed that the combination of video and specialty training does not improve the development or maintenance of medical students' suture and knot tying surgical skills compared to video material training alone (Nousiainen et al., 2008).

It is not enough to study on online education models in the literature. This inadequacy caused experts to oppose videobased education. When we examine the studies carried out in this field, we came across different results. In our study, video-based training has a statistically significant effect on knot tying. The low level of excitement of students is the surprising and thought-provoking result of this study. The fact that the group performing thyroid examination has more confidence in itself than the knot tying group has once again demonstrated the importance of expert supervision in practical training. When we look at the results of our study, we think that video-based education is necessary in the future of medical education. The fact that the COVID-19 pandemic will increase belief and support for video-based education is inevitable.

\section{Conflict of Interest}

The author declares that there is no conflict of interest.

\section{References}

1. Armstrong, A.W., Alikhan, A., Cheng, L.S., Schupp, C., Kurlinkus, C., Eisen, D.B., 2010. Portable video media for presenting informed consent and wound care instructions for skin biopsies: a randomized controlled trial. Br. J. Dermatol. $163,1014-1019$.

2. Armstrong, A.W., Kim, R.H., Idriss, N.Z., Larsen, L.N., Lio, P.A., 2011. Online video improves clinical outcomes in adults with atopic dermatitis: a randomized controlled trial. J. Am. Acad. Dermatol. 64, 502-507.

3. Edwards, M.J., Hannah, K.J., 1985. An examination of the use of interactive videodisc cardiopulmonary resuscitation instruction for the lay community. Comput. Nurs. 3, 250 -252.

4. Fabius, D.B., Grissom, E.L., Fuentes, A., 1994. Recertification in cardiopulmonary resuscitation: a comparison of two teaching methods. J. Nurs. Staff. Dev. 10, 262-268.

5. Fried, G., Feldman, L., Vassiliou, M., Fraser, S., Stanbridge, D., Ghitulescu, G., Andrew, C.G., 2004. Proving the value of simulation in laparoscopic surgery. Ann. Surg. 240, 518-528.

6. Greenhalgh, T., 2001. Computer assisted learning undergraduate education. BMJ. 322, 40-44.
7. Keane, D.R., Norman, G.R., Vickers, J., 1991. The inadequacy of recent research on computer-assisted instruction. Acad. Med. 66, 444-448.

8. Lynch, T.G., Steele, D.J., Johnson Palensky, J.E., Lacy, N.L., Duffy, S., 2001. Learning preferences, computer attitudes, and test performance with computer-aided instruction. Am. J. Surg.181, 368 .

9. Nousiainen, M., Brydges, R., Backstein, D., Dubrowski, A., 2008. Comparison of expert instruction and computer-based video training in teaching fundamental surgical skills to medical students. Surgery.143, 539-544.

10. Orringer, J.S., Fendrick, A.M., Trask, P.C., Bichakjian, C.K., Schwartz, J.L., Wang, T.S., Karimipour, D.J., Johnson, T.M., 2005. The effects of a professionally produced videotape on education and anxiety/distress levels for patients with newly diagnosed melanoma: a randomized, prospective clinical trial. J. Am. Acad. Dermatol. 53, 224-229.

11. Pusic, M.V. 1998. Pediatric residents: are they ready to use computeraided instruction? Arch. Pediatr. Adolesc. Med.152, 494.

12. Prystowsky, J.B., Hassan, M.B., Nahrwold, D.L., 1996. Use of notebook computers for third-year surgery students. Surgery. 120, 304- 307.

13. Satava, RM., 1993. Virtual reality surgical simulator: the first steps. Surg Endosc. 7, 203-205.

14. Schmidt, R.A., Young, D.E., Swinnen, S., Shapiro, D.C., 1989. Summary knowledge of results for skill acquisition: support for the guidance hypothesis. J. Exp. Psychol. Learn. Mem. Cogn. $15,352-359$.

15. Schmidt, R.A., Bjork, R.A., 1992. New conceptualizations of practice: common principles in three paradigms suggest new concepts for training. Psychol. Sci. 3, 207-217.

16. Seymour, N., Gallagher, A., Roman, S., O’Brien, M., Bansal, V., Andersen, D., Satava, R.M., 2002. Virtual reality training improves operating room performance. Ann. Surg. 236, 458464.

17. Shukla, A.N., Daly, M.K., Legutko, P., 2012. Informed consent for cataract surgery: patient understanding of verbal, written, and videotaped information. J. Cataract Refract. Surg. 38, 8084.

18. Summers, A.N., Rinehart, G.C., Simpson, D., Redlich, P.N., 1999. Acquisition of surgical skills: a randomized trial of didactic, videotape, and computer-based training. Surgery. 126, 330-336. 